\title{
Observation of the microstructure of the metastable Ti15Mo alloy after ageing
}

\author{
M. Janeček ${ }^{1, *}$, K. Bartha ${ }^{1}$, M. Cieslar ${ }^{1}$, P. Minárik ${ }^{1}$, J. Stráský $^{1}$ \\ ${ }^{1}$ Department of Physics of Materials, Charles University, Prague, Czech Republic \\ * corresponding author: email - janecek@met.mff.cuni.cz
}

\begin{abstract}
Omega phase particles can be observed by conventional SEM. Metastable Ti15Mo alloy was annealed at 500 ${ }^{\circ} \mathrm{C}$ for 16 hours. During annealing, $\omega$ phase particles grew and got chemically stabilized by expelling Mo atoms. As the result, these ellipsoidal particles, approx. $100 \mathrm{~nm}$ long and $50 \mathrm{~nm}$ wide can be observed using back-scattered electrons signal in conventional SEM due to chemical contrast. TEM study proved that these particles indeed belong to $\omega$ phase. Co-existence of $\beta, \omega$ and $\alpha$ phases was observed. Thin $\alpha$ lamellae were observed along with distorted $\omega$ phase particles by TEM.
\end{abstract}

\section{Introduction}

Since the discovery of the $\omega$ phase in Ti alloys by Frost et al. [1], there have been numerous studies on its formation mechanism and its properties [2]. In the studied Ti15Mo alloy, the structure of $\omega$ phase is hexagonal belonging to the $\mathrm{P} 6 / \mathrm{mmm}$ space group [3]. $\omega$ phase is formed by reversible displacive shuffle transformation [4] and referred to as $\omega$ athermal $\left(\omega_{\text {ath }}\right)$. During ageing, $\omega$ phase grow by irreversible diffusion assisted mechanism [5] and is then referred to as $\omega$ isothermal $\left(\omega_{\text {iso }}\right)$. $\omega$ particles are coherent with $\beta$ matrix and small (a few nanometres to a few tens of nanometres) [6]. Direct observation of $\omega$ phase particles is limited to transmission electron microscopy (TEM) due to their small size $[7,8]$.

$\omega$ phase particles affect subsequent precipitation of $\alpha$ phase during annealing $[9,10]$. Exact mechanism of the $\omega$ assisted nucleation of $\alpha$ phase is not fully resolved and probably differs for different alloys and annealing treatments. Main proposed mechanisms include direct nucleation of $\alpha$ phase from $\omega$ particles $[11,12]$ and nucleation of $\alpha$ phase in some distance from $\omega$ particles due to enrichment of $\beta$ matrix by $\alpha$ stabilizing elements $(\mathrm{Al}$ or $\mathrm{O})$ repulsed from $\omega$ phase [13-15].

Present study on Ti15Mo in this manuscript cannot undoubtedly decide about the exact $\omega$ assisted $\alpha$ precipitation mechanism, however, it provides detailed TEM study which shows co-existence of $\beta, \omega$ and $\alpha$ phases. Recent study by Zháňal [16] proved that during linear heating of Ti15Mo alloy with the heating rate of $5{ }^{\circ} \mathrm{C} / \mathrm{min}$, $\omega$ phase disappears abruptly at $560{ }^{\circ} \mathrm{C}$ by dissolving back to $\beta$ matrix. At $580{ }^{\circ} \mathrm{C}$ material consists of $\beta$ phase only and $\alpha$ phase particles precipitate later at higher temperatures. Situation is very different during isothermal annealing, which provides sufficient time for diffusion controlled growth of $\omega$ particles and simultaneously diffusion controlled $\alpha$ phase precipitation. Co-existence of $\beta, \omega$ and $\alpha$ phases is then possible [17].

\section{Experimental materials and methods}


The Ti15Mo alloy used in this study was supplied from Carpenter Co. in a form of rod with diameter approx. $10 \mathrm{~mm}$. The material was solution treated in an inert Ar atmosphere at the temperature of $810^{\circ} \mathrm{C}$ for 4 hours and subsequently quenched in water. Cylindrical sample with thickness approx. $2 \mathrm{~mm}$ were aged at $500{ }^{\circ} \mathrm{C}$ for 16 hours in a preheated molten salt bath in order to avoid contamination from air. The sample was finally water quenched.

Sample for SEM observation was grinded by emery papers from 320 to 2400 mesh. The sample was subsequently polished by vibratory polishing in three steps - using alumina $(0.3 \mu \mathrm{m})$, alumina $(0.05 \mu \mathrm{m})$ and colloidal silica. Each polishing step took around 8 hours. The sample was carefully cleaned from colloidal silica by water and ethanol. Careful polishing is critical for successful SEM observations.

Zeiss Auriga CrossBeam at acceleration voltage of $10 \mathrm{kV}$ was used for SEM analysis. The lamella for TEM observation was prepared using Ga focused ion beam (FIB) in the SEM. The TEM study was performed on JEOL 2200 FS transmission electron microscopy using acceleration voltage of $200 \mathrm{kV}$.

\section{Results and discussion}

Figure 1 shows the SEM - BSE micrograph of the Ti15Mo alloy after ageing $500{ }^{\circ} \mathrm{C} / 16$ hours. Image shows interior of a $\beta$ grain with two morphologically different types of precipitates. Elongated lamellae could be clearly attributed to $\alpha$ phase, however, observation of small $(\sim 100 \mathrm{~nm}$ size $)$ ellipsoidal particles was rather surprising. TEM study provided below proves that these small ellipsoidal particles are indeed $\omega$ particles. To our knowledge, this is the first observation of $\omega$ phase particles by standard SEM. The contrast in the backscattered electrons (BSE) image in Figure 1 is given by Z-contrast (chemical contrast). $\omega$ phase particles are observable due to repulsion of heavier Mo atoms to $\beta$ matrix. Such repulsion was quantified previously with the use of advanced method of 3D atom probe tomography [18]. As the result of Mo depletion, $\omega$ phase particles appear darker in SEM-BSE, similarly to $\alpha$ phase. SEM allows distinguishing between $\alpha$ and $\omega$ only in terms of morphology, direct proof is possible by electron diffraction in TEM.

Observation of $\omega$ phase particles by SEM is possible due to two factors (except specimen preparation): $\omega$ particles are comparatively big (their size is around $100 \mathrm{~nm}$ ) and strongly chemically stabilized by Mo depletion. Both these factors were achieved by long-term annealing $(16 \mathrm{~h})$ at $500{ }^{\circ} \mathrm{C}$. This temperature is below the solvus temperature of $\omega$ phase and, despite $\alpha$ phase precipitation, not all $\omega$ particles were consumed by stable $\alpha$ phase.

$\alpha$ phase lamellae are clearly thin (arguably less than $100 \mathrm{~nm}$ ) and in many cases very long - several micrometers. Note that apparent thickness of a lamellae depends on its orientation with respect to the sample surface. Indistinct gray contrast in some areas is caused by lamellae that are almost parallel to the sample surface and the contrast is blurred due to the penetration depth of electrons exceeding the width of the lamellae - width of the surface layer from which the BSE signal is acquired can reach few hundreds nm and correlates with acceleration voltage. 


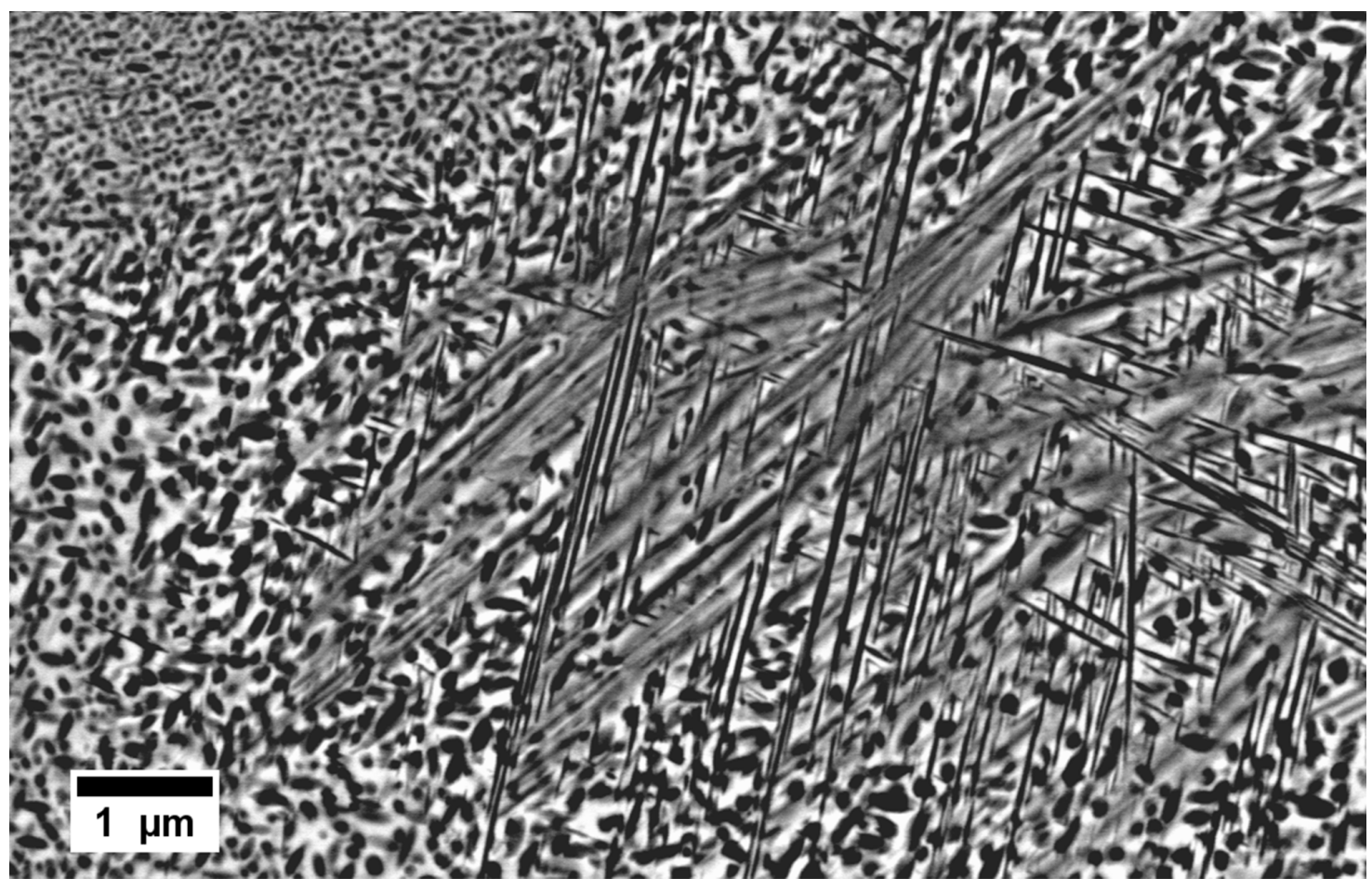

Figure 1. SEM micrograph of the Ti15Mo alloy after ageing at $500^{\circ} \mathrm{C}$ for 16 hours

A TEM lamella containing particles of both $\omega$ phase and $\alpha$ phase was prepared by FIB in SEM, carefully thinned and used for TEM analysis. Figure 2(a) shows the TEM bright field (BF) image of the $\beta$ matrix with $\omega$ precipitates with typical ellipsoidal shape. The longer axis is aligned with one of $[111]_{\beta}$ directions of $\beta$ matrix. The size of particles along this longer axis exceeds $100 \mathrm{~nm}$, in perpendicular direction the size can reach $60 \mathrm{~nm}$. The aspect ratio is approximately 2:1 for all particles. Note, that all of the $\omega$ particles, which are in diffraction contrast, are elongated in one specific direction which is associated with the given crystallographic orientation of the $\omega$ lattice with respect to the $\beta$ matrix. $\omega$ particles in different orientations (different families of the $\omega$ particles) cannot be resolved in BF image as they are not in diffraction contrast. The selected area diffraction (SAED) pattern of zone axis of $[411]_{\omega}$ from one of the $\omega$ particle is displayed in Figure 2(b) proving that observed particles are indeed $\omega$ phase particles [19]. 

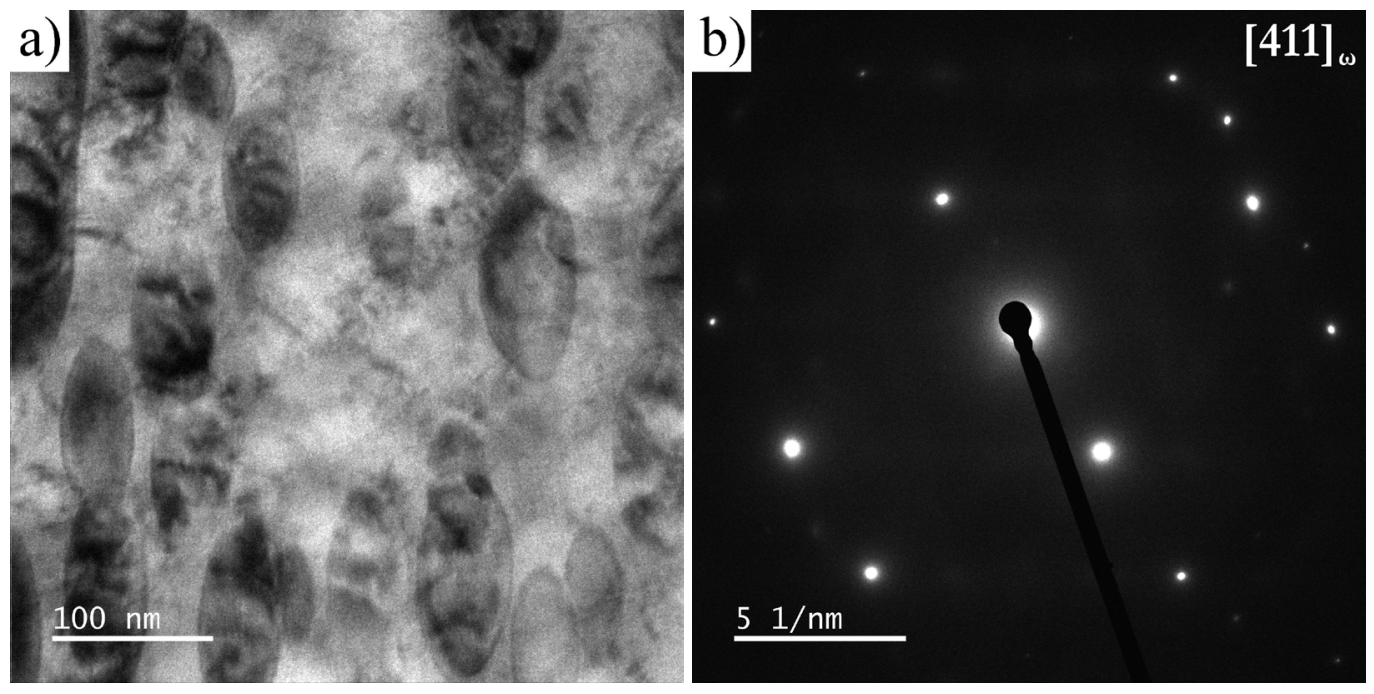

Figure 2. (a) TEM bright field images of the $\omega$ phase and (b) SAED pattern from one $\omega$ phase particle in zone axis of $[411]_{\omega}$

Figure 3(a) shows the SAED pattern from the interior of the $\beta$ grain in zone axis of [315] $]_{\beta}$. The dark field (DF) image from one of the $\omega$ reflection (highlighted by red circle in Figure 3(a)) is shown in Figure 3(b). $\omega$ particles elongated in one direction are clearly visible. Note that TEM lamella is thicker than individual $\omega$ particles and therefore overlapping of particles may occur.

TEM micrographs in Figures 3(c) and 3(d) show region with precipitated $\alpha$ lamellae. Figure 3(c) is dark field (DF) image using the $\omega$ phase reflection highlighted in the SAED pattern in Figure 3(a). Therefore, $\omega$ phase particles appear bright. These particles are distorted and we argue that this distortion is caused by the proximity of $\alpha$ lamellae. Figure 3(d) shows bright field image of the same area showing $\alpha$ lamellae in white with distorted $\omega$ phase particles in between of these lamellae. The apparent thickness of $\alpha$ lamellae is around $100 \mathrm{~nm}$, but this value cannot refer to the true thickness of $\alpha$ lamellae because observed thickness depends on the orientation of lamellae with respect to the sample surface. Note again that thickness of TEM foil causes overlapping of $\omega$ particles and $\alpha$ lamellae.

The most important finding from Figures 3(c) and 3(d) is that $\omega$ phase particles and $\alpha$ phase lamellae co-exist very closely to each other. We assume that initial stage of $\alpha$ phase precipitation is captured in the image and $\omega$ phase particles would be consumed by growing $\alpha$ lamellae during further annealing.

In $\beta$ matrix, $\alpha$ phase precipitates in the form of lamellae in order to reduce elastic distortion and create a coherent interface [20]. Moreover, the lamellar morphology satisfies the Burgers orientation relation and may indicate low interfacial energy [21]. Presented observations show that $\alpha$ phase is formed with lamellar morphology also in presence of $\omega$ phase particles. Exact mechanism of $\alpha$ phase precipitation cannot be inferred from the TEM imaging, but the TEM analysis proved that $\omega$ phase particles are observable by SEM. 
The advantage of SEM observations is that it is less-local method than TEM. Especially in-situ SEM observation during annealing may provide valuable information about the mechanism of $\alpha$ phase precipitation in presence of $\omega$ phase particles. Note that in-situ TEM is not possible due to thin foil effect ( $\omega$ and $\alpha$ particles do not evolve in TEM foil) [22].
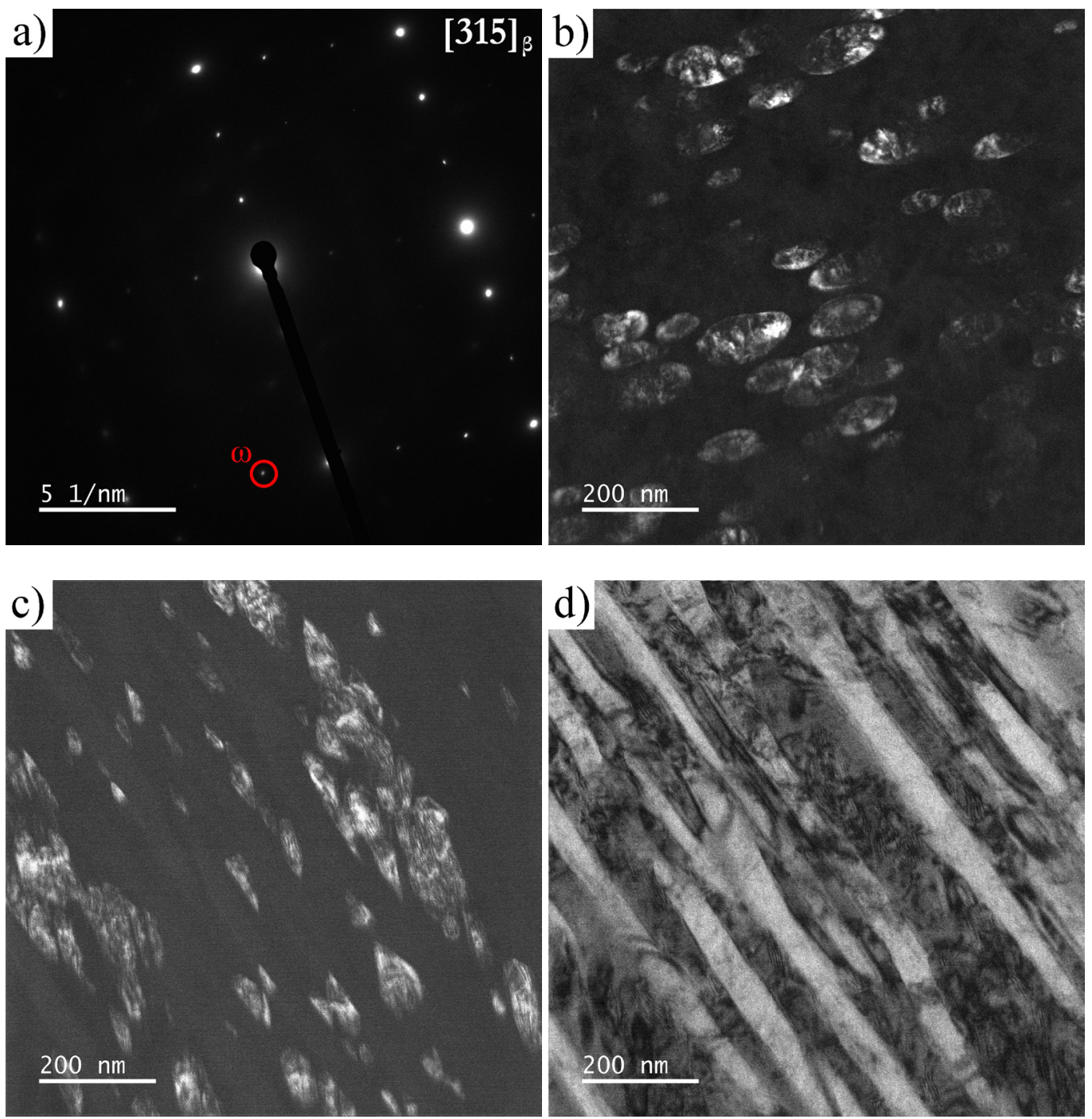

Figure 3. (a) SAED pattern recorded from aged Ti15Mo alloy in zone axis of $[315]_{\beta}$ and with highlighted $\omega$ phase reflection (b) corresponding dark field image from the $\omega$ phase reflection in the interior of the $\beta$ grain, (c) corresponding dark field image from the $\omega$ phase reflection in the vicinity of $\alpha$ lamellae, (d) bright field image showing $\alpha$ lamellae bright with distorted $\omega$ phase particles in diffraction contrast 


\section{Conclusions}

Ti15Mo alloy was annealed at $500{ }^{\circ} \mathrm{C}$ for 16 hours. Co-existence of $\beta, \omega$ and $\alpha$ phases was observed by conventional SEM and proven by TEM analysis. Ellipsoidal particles of $\omega$ phase exceed $100 \mathrm{~nm}$ in length and $50 \mathrm{~nm}$ in width. Thin $\alpha$ lamellae are observed in close vicinity to distorted $\omega$ phase particles.

\section{References}

[1] P. D. Frost, W. M. Parris, L. L. Hirsch, J. R. Doig, C. M. Schwartz. Isothermal transformation of titanium-chromium alloys. Trans. ASM, 46 (1954) 231-256

[2] S. Banerjee and P. Mukhopadhyay. Phase transformations: examples from titanium and zirconium alloys, Volume 12 (2010), Elsevier

[3] J. M. Silcock, M. H. Davies, and H. K. Hardy. Structure of the $\omega$-precipitate in titanium-16\% vanadium alloy. Institute of Metals. Monograph and report series 18 (1955) 93-105

[4] D. De Fontaine. Mechanical instabilities in the b.c.c. lattice and the beta to omega phase transformation. Acta Metallurgica 18 (1970) 275-279

[5] T. W. Duerig, G. T. Terlinde, and J. C. Williams. The $\omega$-phase reaction in titanium alloys. Titanium ' 80 Science \& Technology - Proceedings of the 4th International Conference on Titanium, 2 (1980) 1299-1308

[6] J. C. Williams. Critical review: Kinetics and phase transformations. Titanium science and technology, 3 (1973) 1433-1494.

[7] E. Sukedai, H. Hashimoto, M. Hida, Fine Structure of As-Quenched Omega-Phase of Ti-Mo Alloy Observed by HREM with Image Processing. Japanese Journal of Applied Physics 26 (1987)

[8] A. Devaraj, S. Nag, R. Srinivasan, R.E.A. Williams, S. Banerjee, R. Banerjee, H.L. Fraser, Experimental evidence of concurrent compositional and structural instabilities leading to $\omega$ precipitation in titaniummolybdenum alloys, Acta Materialia 60 (2012) 596-609

[9] Y. Zheng, R. Williamd, D. Wang. R. Shi, S. Nag, P. Kami, J. M. Sosa, R. Banerjee, Y. Wang, H. Fraser, Role of $\omega$ phase in the formation of extremely refined intragranular $\alpha$ precipitates in metastable $\beta$-titanium alloys. Acta Materialia 103 (2016) 850-858.

[10] N. G. Jones, R. J. Dashwood, M. Jackson, D. Dye, $\beta$ Phase decomposition in Ti-5Al-5Mo-5V-3Cr. Acta Materialia 57 (2009) 3830-3839.

[11] J. C. Williams, M. J. Blackburn, The influence of misfit on the morphology and stability of the omega phase in titanium-transition metal alloys. Transactions of the Metallurgical Society of AIME 245 (1969) $2352-2355$

[12] F. Prima, P. Vermaut, G. Texier, D. Ansel, and T. Gloriant. Evidence of $\alpha$-nanophase heterogeneous nucleation from $\omega$ particles in a $\beta$-metastable Ti-based alloy by high-resolution electron microscopy. Scripta Materialia, 54 (2006) 645-648 
[13] S. Azimzadeh and H. J. Rack. Phase transformations in Ti-6.8 Mo-4.5Fe-1.5 Al. Metallurgical and Materials Transactions A, 29 (1998) 2455-2467

[14] S. Nag, R. Banerjee, R. Srinivasan, J. Y. Hwang, M. Harper, and H. L. Fraser. $\omega$-assisted nucleation and growth of $\alpha$ precipitates in the Ti-5Al-5Mo-5V-3Cr-0.5Fe $\beta$ titanium alloy. Acta Materialia, 57 (2009) 21362147

[15] T. Li, D. Kent, G. Sha, M. S. Dargusch, and J. M. Cairney. The mechanism of $\omega$-assisted $\alpha$ phase formation in near $\beta$-Ti alloys. Scripta Materialia, 104 (2015) 75-78

[16] P. Zháňal, P. Harcuba, M. Hájek, B. Smola, J. Stráský, J. Šmilauerová, J. Veselý, M. Janeček, Evolution of $\omega$ phase during heating of metastable $\beta$ titanium alloy Ti-15Mo. Journal of Materials Science 53 (2018) $837-845$

[17] M. Sabeena, Alphy George, S. Murugesan, R. Divakar, E. Mohandas, M. Vijayalakshmi,, Microstructural characterization of transformation products of bec $\beta$ in Ti-15 Mo alloy, Journal of Alloys and Compounds 658 (2016) 301-315

[18] A. Devaraj, R.E.A. Williams, S. Nag, R. Srinivasan, H.L. Fraser, R. Banerjee, Three-dimensional morphology and composition of omega precipitates in a binary titanium-molybdenum alloy, Scripta Materialia 61 (2009) 701-704

[19] A. George, V. Sharma, R. Divakar, M. Sabeena, E. Mohandas, Transmission electron microscopy studies and modeling of 3D reciprocal space of $\omega$ forming alloy, Micron 102 (2017) 73-87

[20] D. A. Porter, K. E. Easterling, and M. Y. A. Sherif, 'Phase Transformations in Metals and Alloys (Revised Reprint) Third Edition', CRC Press, 1981

[21] T. Furuhara, T. Makino, Y. Idei, H. Ishigaki, A. Takada, and T. Maki, 'Morphology and Crystallography of $\alpha$ Precipitates in $\beta$ Ti-Mo Binary Alloys', Mater. Trans. JIM 39 (1998) 31-39

[22] K. Bartha, Phase transformations in ultra-fine grained titanium alloys, Doctoral Thesis, Charles University, 2019 